\title{
Providing Multimedia QoS Methods over Software Defined Networks: A Comprehensive Review
}

\author{
Mohammad Reza Parsaei, Reza Javidan, Ali Fatemifar, Sina Einavipour \\ Department of Computer Engineering and IT, \\ Shiraz University of Technology, \\ Shiraz, Iran
}

\begin{abstract}
In recent years, numerous claims on non-responsiveness of architecture of current networks and Internet to users' daily demands have been raised. To deal with the problem, new architectures have been suggested. Software-defined network (SDN) introduced a new architecture for computer network in which control level and data transfer level are separated from each other. This architecture has contributed to smartness, flexibility, and controllability of computer networks. Among advantages of this architecture, one could point to easy control and isolation of different streams without being concerned with implementation and test of newly suggested protocols and architectures on real networks. In this paper, introduction of new network architecture is followed by detailing different methods for guaranteeing the quality of service (QoS) in software-defined network and elaborating the challenges ahead. In fact, the objective of present paper is to represent the effect of use of software-defined network on quality of service in different multi-media applications and based on the architecture of the networks in which control layer and directing layer are isolated. Such an architecture enables the use of different routing algorithms for different streams. In the case of these methods, the introduced methods are applied for improving the use of video streams.
\end{abstract}

\section{Keywords}

Quality of Service, Multimedia, Future Internet Architecture, Software Defined Networks.

\section{INTRODUCTION}

The use of Internet in an extensive scale has turned it into a part of critical infrastructure of our society. Concurrent development of physical infrastructure and protocols and efficiency of the Internet is a very difficult task. The need for applications and online services is increasing and it is essential to see that Internet is able to evolve so as to address the new challenge. This is while network setting in industries and university are limited to specific solutions with few alternatives with very slow progress [1].

One of the solutions that is paid attention to today is SDN. SDN isolates network control from data transfer. As a result, routing algorithms, filtering, firewalls and other algorithms used in different elements of the network are transferred to controller and switches solely transfer the data. With help of this architecture, networks become smarter and more controllable, their dependence on hardware is reduced and their management will become more flexible and simpler. In recent decades, the use of multi-media services in Internet has increased significantly. It is predicted that video traffic will constitute over 90 percent of Internet traffic during next few years [2].
During past decades, Internet Engineering Task Force (IETF) had developed different QoS architectures but none of them have been definitively successful or implemented in global scale. The reason behind such failure is that QoS architectures such as IntServ [3] and DiffServ [4] use distributed routing architecture because such architectures do not offer a general viewpoint of network resources. In the case of using SDN, the routes of delivering video streams could operate based on change of network conditions through dynamic re-routing and forwarding stream in a route with lowest level of congestion. To do this, numerous algorithms have been suggested for multimedia forwarding through software-defined network. In this paper, the most significant suggested algorithms and their comparisons are reviewed.

The objective of present paper is to show the effect of using QoS software-defined networks on multimedia uses. The effect is represented by attention to architecture of these networks in which control layer and directing layer are separate. This characteristic enables the use of different routing algorithms for different streams. The methods introduces for such networks have been generally used for improved use of video streams.

The present paper is organized in the following manner. Section II introduces the structure of SDNs and their multimedia applications. Section III details the suggested methods for improving quality of service in the case of multimedia applications. Finally, section VI concludes the paper and offers a short comparison between best methods introduced hitherto.

\section{STRUCTURE OF SOFTWARE DEFINED NETWORKS}

The software-defined networking are recognized as a method for enhancing the programming capability of computer networks. The architecture of such networks isolates control layer from data transfer layer of devices on the networks and therefore, it enables development of new protocols of routing and directing without requiring changes of network hardware. One of the common implementations of software-defined networks is implementation based on open standard of OpenFlow. In such networks, the nodes are called OpenFlow switches which are responsible for directing. This is while routing decisions and control functions are allocated to a central element called "controller". Structure of Software Defined Networks and components are presented in Figure 1. 


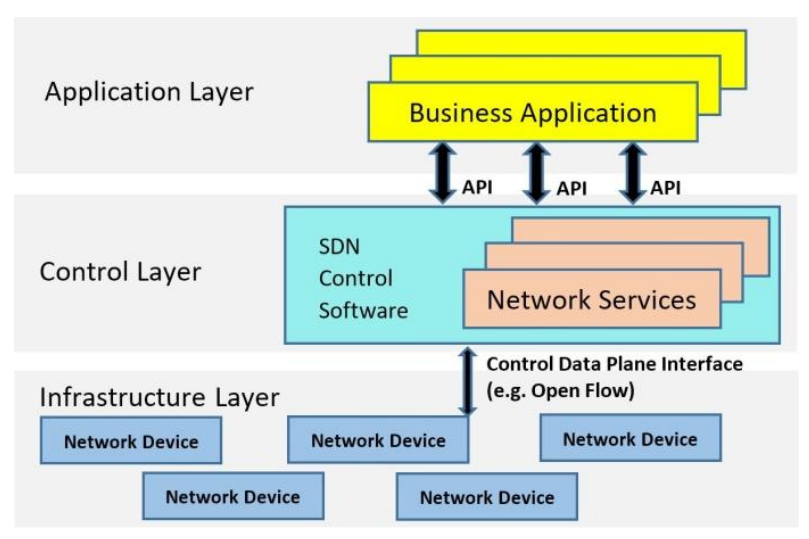

Fig 1: Structure of Software Defined Networks

As shown in figure 1, QoS network architecture is composed of three layers of infrastructure, control and application. The controller in which network smartness is concentrated is in control layer. The primary task of controller is decision on driving intra-network streams and filling the routing tables of underlying layer. Controller could be regarded as an operating system which provides a programmatic interface for the network and applications could conduct security tasks on it. Among such tasks, one could point to control and application of different policies and management tasks such as metering, monitoring, resource provision and new capabilities.

In addition to the three layers mentioned before, one could point to southbound and northbound interfaces. The southbound interface relates the control layer to infrastructure. With such interface, the controller will be able to discover network topology, develop different reports of network switches, managing and issuing different orders to them and responding to request of applications regarding network streams. The protocols OpenFlow, extensible messaging and presence protocol (XMPP) [5], one platform kit (OnePK; Cisco Company) and path computation element communication protocol (PCEP) [6] could be used as southbound interface. Among such protocols, OpenFlow is the most famous one.

By using northbound interfaces, applications and management systems extract their required information on network and streams from controller. Among northbound interfaces, one could point to Frenetic [7], FML [8], Nettle [9], OSGi [10], ALTO [11] and Restful API.

The current networks act based on best effort basis and it cannot provide for traffic of multimedia traffic. The traffic requires reliable network resources and it can tolerate definite levels of delay, jitter and packet loss. In recent few decades, numerous methods have been suggested for satisfying quality of service for multimedia applications. However, none of them succeeded completely. Among such methods, one could point to IntServ and DiffServ mechanisms which guarantee quality of service by reserving necessary resources and scheduling techniques respectively. Among such problems, one could point to lack of a general viewpoint of network and selection of route in a node-to-node manner. As a result, they cannot lead to optimal route. In addition, implementation of such methods requires changes of hardware in network devices. In software-defined networks, control and data transfer are separate and this contributes to flexibility of network control but reduces dependence on hardware. Therefore, projects for improvement and optimization of multimedia quality are done through software-defined networks as detailed in the next sections. It should be noted that most of these projects have been done for improving the traffic of video streaming.

\section{LITERATURE REVIEW}

Civanlar [12] introduced an OpenFlow architecture for guaranteeing the quality of multimedia services which use scalable video encoding. The traffics of scalable video encoding is composed of a main layer and some incremental layers. The incremental layers of decoder are developed based on the main layer. That is why main layer packets should be lost during transfer or be transferred erroneously. In this architecture, concurrent controller conducts two routings on network traffic. The first routing is called "quality of service routing" and streams of main traffic layers of scalable video encoding follow it. The second routing is called "best effort" which is done based on shortest path algorithm and incremental layers are driven based on it. The routing of quality of service is done based on minimization of weighted sum of traffic route length of quality of service, amount of packets lost in normal traffic, and supposition of zero number of packs lost during traffic (including quality of service). Therefore, QoS routing could affect other traffics such as best effort traffic and increase the amount of packets lost for the traffic.

Egilmez [13] analyzed routing algorithm of the traffic in two different scenarios. The first scenario is related to the time when main layer of scalable video encoding traffic is recognized as a traffic which is characterized by quality of service and no missing packet. The second scenario refers to the time when incremental layers are recognized as QoS traffic. The difference is that second scenario recognizes the possibility of packet loss. The scenario was implemented and evaluated through network optimization library of LEMON. Based on previously conducted evaluations, the first scenario is 14 percent more efficient that best-effort routing while second scenario is 6.5 percent more efficient that the first scenario.

Laga [14] prioritized different layers of scalable video encoding stream and implemented QoS routing through change of Dijkstra algorithm so as to improve this type of video streams. In this method, weight of each link is determined based on a mixed and weighted function of link delay and remaining link bandwidth. This method is implemented by extending Beacon controller and assessed through Mininet simulator. The results suggest that the method reduces delay of delivery of packets up to 72 percent in the main layer, 48 percent in first secondary layer, and 8 percent in second secondary layer. In addition, the method increased delay of third secondary layer up to 66 percent when compared with shortest path method.

Karl [15] changed the metric model of Dijkstra algorithm to improve quality of service in the network. The paper suggests certain methods for measuring network parameters such as rate of packet loss, delay and bandwidth of each link. In order to measure packet loss rate, the number of forwarded packets (tx) on each port $\mathrm{p}$ which is stored by OpenFlow switch is used. The equation 1 and 2 show the means of packet loss rates through $\mathrm{N}$ samples in the switch and link $\mathrm{L}$ :

$N_{t x \mid r x, \text { mean }}(n, p)=\frac{\sum_{k=1}^{N_{\text {Sample }}} N_{t x \mid r x}^{k}(n, p)}{N_{\text {Sample }}}$ 


$$
\operatorname{PLR}(l)=\frac{N_{t x, \text { mean }}(\operatorname{src}(l))-N_{r x, \text { mean }}(d s t(l))}{N_{t x, \text { mean }}(\operatorname{src}(l))}
$$

In order to measure link delay, a UDP packet is forwarded from controller to switch of a link header. The switch forwards the packet to the switch of another link header and second switch returns the packet to controller. In the case of reducing the time for forwarding and delivery of packet to controller, relative link delay will be determined. In addition, determining the difference between forwarded and received bytes in p-switch port in a definite time and dividing the difference by the duration result in determining bandwidth of each switch. At the end, the paper presumes fixed packet loss rate and link delay and uses the consumed bandwidth as Dijkstra metric so as to compare the routing and shortest path routing of software-defined networks through NOX controller on RTP/RTSP traffic.

Kim [16] introduced a framework of automatic service quality control in converged networks. In the paper, traffic shaping or static queuing was used. The controller is able to allocate traffics of different applications to network slices and fulfill qualitative requirements of the slides dynamically. In this case, network administrators should solely define a high-level slice specification or each service or client. Then, they reserve automatic network resource controller for satisfaction of efficiency. In order to achieve flexibility, every description of individual stream is defined based on requirements of a client as sum of streams of a traffic or a combination of them. In order to evaluate controller, 4 types of traffics were used. Three types of the traffic is quality sensitive and the remaining type is insensitive. There are three sensitive traffics on UDP. When insensitive traffic exists on UDP, packet loss is observed on it. However, the other three traffics maintain their expected efficiency. When insensitive traffic exists on TCP, no packet loss is found.

Owens [17] drew upon IntServ architecture to design a SDNbased architecture called Video over SDN. Owens attempted to address the problems of IntServ architecture. One of the main problems of IntServ is its dependence on routing protocol used in the network as well as relevant route selection. In VSDN, a routing module is introduced which could opt for the best method based on requirement of video stream application. The selection is done by considering centrality of controller and its ability to measure QoS parameters. In the paper, a prototype of suggested architecture is developed based on characteristics of OpenFlow 1.3.1 and number of exchanged messages in the system is solely examined. The results suggested that increase in number of network nodes was followed by linear increase in number of messages.

Georgopoulos [18] implemented a framework in which controller allocates proper bandwidth to the stream based on characteristics of video stream on-demand devices (i.e. tablet, mobile phone, etc.) and characteristics of on-demand video stream (i.e. bit rate, video time). Therefore, Georgopoulos attempted to improve quality of experience of any type of client.
Jivorasetkul [19] introduced compression in traditional networks. In such networks, compression is done in a node-tonode manner and for all switches and routers in the path. This led to increased delay for the packet to get its destination. In the paper, OpenFlow switches are used to suggest an end-toend method of compression. In this method, following header compression the terminal hosts allocate an ID to the packet based on fixed data of closed header. In this case, the packet at terminal hosts are identified based on ID and routing of the packet is done based on headers of second layer and without requirement of decompression. The method was not implemented and evaluated.

Kassler [20] suggested an architecture for guaranteeing QoE for a video conferencing. The QoS elements of matching and optimization function [21] and path assignment function are among main elements of the architecture. QMOF is located in application layer of software-defined networks and it supports optimal delivery of session between two users and determination of intended parameters for establishing meeting service. Then, media degradation path is determined based on such parameters. PAF is located in control layer of softwaredefined networks. Based on reported MDP, RAF computes the optimal path for each stream. This idea is originally taken from NGN networks and this architecture was not implemented too.

\section{REVIEW AND DISCUSSION}

Scalable video codec is used for all of the solutions. In this type of codec, video is composed of a main layer and a number of incremental layers. As a result, for streams of each layer different routing methods and different resource allocation policies were used and this was enabled by using software-defined networks and isolation of control layer from driving layer. In this architecture, depending on type of stream and requirement, controller could provide different routing algorithms for network drivers. Consequently, different video layers are routed differently and based on the requirement. In scalable video codec, the main layer is the most significant one and any case of pack loss for such a layer is acceptable. This is while incremental layers improve the quality of received video. Therefore, in all of the solutions the priority of using resources is given to the main layer and major difference of solutions lies in type of routing and driving incremental layers.

Based on evaluations of algorithms detailed in the present paper, one could conclude that all of the solutions involving software-defined networks contribute to quality of service significantly. The effect of software-defined networks on quality of service of multimedia applications is inevitable. Considering the adopted routing methods and metrics used in each of them, one may infer that Kim's method is suitable for homogenous networks. In applications in which a user is using a device (i.e. tablet, mobile phone, laptop, etc.) and making decisions on use of different routing methods for intended layers is needed, one may use the method that Georgopoulos suggested. In addition, for use of multimedia in video conferencing the method suggested by Kassler could be adopted. Table 2 offers a comparison between the most significant methods suggested in the present paper. 
Table 1. Comparison between Methods

\begin{tabular}{|c|c|c|c|c|c|}
\hline Title of Paper & Solution & Controller & $\begin{array}{c}\text { Type of } \\
\text { Implementation }\end{array}$ & OpenFlow & Result \\
\hline $\begin{array}{l}\text { A QoS-Enabled } \\
\text { OpenFlow } \\
\text { Environment for } \\
\text { Scalable Video } \\
\text { Streaming. }\end{array}$ & $\begin{array}{l}\text { Use of QoS routing for } \\
\text { stream of main layer } \\
\text { traffic and routing the } \\
\text { best effort for traffic of } \\
\text { incremental layers }\end{array}$ & NOX & Simulation & Version 1.0 & $\begin{array}{l}\text { Increase of PSNR in video } \\
\text { to be sent }\end{array}$ \\
\hline $\begin{array}{l}\text { SCALABLE VIDEO } \\
\text { STREAMING } \\
\text { OVER OPENFLOW } \\
\text { NETWORKS:AN } \\
\text { OPTIMIZATION } \\
\text { FRAMEWORK } \\
\text { FOR QOS } \\
\text { ROUTING. }\end{array}$ & $\begin{array}{l}\text { Developing an } \\
\text { optimized architecture } \\
\text { for design of controller, } \\
\text { use of optimal routing } \\
\text { for two types of main } \\
\text { and incremental traffic } \\
\text { and use of shortest } \\
\text { route for other traffics. }\end{array}$ & Floodlight & Simulation & Version 1.0 & $\begin{array}{l}\text { Increase of efficiency to } \\
20.5 \text { percent at most }\end{array}$ \\
\hline $\begin{array}{l}\text { Optimizing Scalable } \\
\text { Video Delivery } \\
\text { Through } \\
\text { OpenFlow Layer- } \\
\text { based Routing. }\end{array}$ & $\begin{array}{l}\text { Prioritization of } \\
\text { different layers of } \\
\text { scalable video encoding } \\
\text { and routing of quality } \\
\text { of service through } \\
\text { change of Dijkstra } \\
\text { algorithm. }\end{array}$ & Beacon & $\begin{array}{l}\text { Simulation } \\
\text { Mininet }\end{array}$ & Version 1.0.1 & $\begin{array}{l}72 \text { percent reduction of } \\
\text { video freeze in high-load } \\
\text { networks }\end{array}$ \\
\hline $\begin{array}{l}\text { Multimedia } \\
\text { Optimized Routing } \\
\text { in OpenFlow } \\
\text { Networks. }\end{array}$ & $\begin{array}{l}\text { Improvement of QoS } \\
\text { by changing metric } \\
\text { model of Dijkstra } \\
\text { algorithm. }\end{array}$ & NOX & Simulation & Version 1.0 & $\begin{array}{l}\text { Improving dynamic routing } \\
\text { and enhancing the quality } \\
\text { of transfer for applications } \\
\text { that are based on } \\
\text { RTSP/RTP stream }\end{array}$ \\
\hline $\begin{array}{l}\text { Automated and } \\
\text { Scalable QoS } \\
\text { Control for Network } \\
\text { Convergence. }\end{array}$ & $\begin{array}{l}\text { Introducing a } \\
\text { framework for } \\
\text { automatic QoS control, } \\
\text { use of static queuing or } \\
\text { traffic formation. }\end{array}$ & NOX & $\begin{array}{l}\text { Simulation and } \\
\text { Experimental }\end{array}$ & Version 1.3 & $\begin{array}{l}\text { Higher efficiency of } \\
\text { resources of homogenous } \\
\text { networks with fabric } \\
\text { topology }\end{array}$ \\
\hline $\begin{array}{l}\text { OpenQoS: An } \\
\text { OpenFlow Controller } \\
\text { Design for } \\
\text { Multimedia Delivery } \\
\text { with End-to-End } \\
\text { Quality of } \\
\text { Service over } \\
\text { Software-Defined } \\
\text { Networks. }\end{array}$ & $\begin{array}{l}\text { Introducing a modular } \\
\text { solution for selecting a } \\
\text { suitable route based on } \\
\text { solution of limited } \\
\text { shortest path problem. }\end{array}$ & Floodlight & Experimental & Version 1.0 & $\begin{array}{l}\text { Integrated delivery of } \\
\text { video with least } \\
\text { disturbance in the case of } \\
\text { using an unreliable transfer } \\
\text { protocol such as UDP, } \\
\text { guaranteeing best-quality } \\
\text { video delivery in the case } \\
\text { of using a reliable transfer } \\
\text { protocol (e.g. TCP) }\end{array}$ \\
\hline $\begin{array}{l}\text { Video over } \\
\text { Software Defined } \\
\text { Networking. }\end{array}$ & $\begin{array}{l}\text { Solving problems of } \\
\text { IntServ architecture } \\
\text { through VSDN. }\end{array}$ & Floodlight & Simulation & Version 1.3 .1 & $\begin{array}{l}\text { Selecting the best route } \\
\text { based on demanded } \\
\text { requirement of } \\
\text { applications. }\end{array}$ \\
\hline
\end{tabular}

\section{CONCLUSION}

In the present paper, architectures and methods for offering the required quality of multimedia applications in current networks are introduced. Then, advantages, problems and reasons of their failures were reviewed. Then, methods implemented in software-defined networks and results of evaluation of their efficiency were detailed. The methods are generally adopted for improving the use of video streams. In video-conferencing streams, the parameters of delay, PSNR and jitter delay parameters offered the highest improvement in the case of adopting shortest path method with queue. In the next position, the shortest path method without queue offered the second best improvement.

More work is needed to support an efficient architecture so that forwarders can autonomously report congestion conditions as opposed to the controller continuously polling the forwarders on the data path of a QoS flow. A simple configuration mechanism is needed to program the forwarder with performance thresholds so that it can generate a notification when the threshold is exceeded. 


\section{REFERENCES}

[1] Rowshanrad, S., Parsaei, M. R. and Keshtgari, M. "IMPLEMENTING NDN USING SDN: A REVIEW ON METHODS AND APPLICATIONS," IIUM Engineering Journal, vol. 17, no. 2, pp.11-20, 2017.

[2] Parsaei, M. R., Khalilian, S. H. and Javidan, R. "A Comparative Study on Fault Tolerance Methods in IP Networks versus Software Defined Networks," International Academic Journal of Science and Engineering. vol. 3, no. 4, pp. 146-154, 2016.

[3] Braden, R., Clark, D. and Shenker, S. "Integrated services in the Internet architecture: an overview," RFC 1633, 1994.

[4] Blake, S., Black, D., Carlson, M., Davies, E., Wang, Z. and Weiss, W. "An architecture for differentiated services,” RFC 2475, 1998.

[5] Saint-Andre, P. "Extensible Messaging and Presence Protocol (XMPP) :Core,” RFC 3920, 2004

[6] Vasseur, J. and Roux, J. "Path computation element (PCE) communication protocol (PCEP)," RFC 5440, 2009.

[7] McKeown, N., Anderson, T., Balakrishnan, H., Parulkar, G., Peterson, L., Rexford, J., Shenker, S. and Turner, J. "OpenFlow : Enabling Innovation in Campus Networks," ACM SIGCOMM Computer Communication, vol. 38, pp. 69-74, 2008

[8] Foster, N., Harrison, R., Freedman, M.J., Monsanto, C., Rexford, J., Story, A. and Walker, D. "Frenetic: a network programming language," in Proceedings of the 16th ACM SIGPLAN international conference on Functional programming (ICFP), pp. 279-291, 2011.

[9] Voellmy, A. and Hudak, P. "Nettle: taking the sting out of programming network routers," in Proceedings of the 13th international conference on Practical aspects of declarative languages (PADL), pp. 235-249, 2011.

[10] Alliance, O. "Osgi service platform, core specification, release 4, version 4.1,” OSGi Specification, 2007.

[11] Seedorf, J. and Burger, E. "Application-layer traffic optimization (ALTO) problem statement," RFC 5693, 2009.

[12] Civanlar, S., Parlakisik, M., Tekalp, A.M., Gorkemli, B., Kaytaz, B. and Onem, E. "A QoS-enabled OpenFlow Environment for Scalable Video Streaming," in Proc of IEEE GLOBECOM Workshops, pp. 351-356, 2010.
[13] Egilmez, H.E., Gorkemli, B., Tekalp, A.M. and Civanlar, S. "Scalable video streaming over OpenFlow networks: An optimization framework for QoS routing," in 18th IEEE International Conference on Image Processing (ICIP), pp. 2241 - 2244, 2011.

[14] Laga, S., Van Cleemput, T., Van Raemdonck, F., Vanhoutte, F., Bouten, N., Claeys, M. and De Turck, F. "Optimizing scalable video deliv ery through OpenFlow layerbased routing," in Network Operations and Management Symposium (NOMS), IEEE, pp. 1-4, 2014.

[15] Karl, M., Gruen, J. and Herfet, T. "Multimedia optimized routing in OpenFlow networks," in 19th IEEE International Conference on Networks (ICON), pp. 1-6, 2013

[16] Kim, W., Sharma, P., Lee, J., Banerjee, S., Tourrilhes, J., Lee, S.J. and Yalagandula, P. "Automated and scalable qos control for network convergence," Proc. INM/WREN, vol. 10, pp. 1-6, 2010.

[17] Owens, I. and Durresi, A. "Video over Software Defined Networking (VSDN)," in 2013 16th International Conference on Network-Based Information Systems (NBiS), pp. 44-51, 2013.

[18] Georgopoulos, P., Elkhatib, Y., Broadbent, M., Mu, M and Race, N. "Towards network-wide QoE fairness using openflow-assisted adaptive video streaming," in Proceedings of the 2013 ACM SIGCOMM workshop on Future human-centric multimedia networking, pp. 1520, 2013.

[19] Jivorasetkul, S., Shimamura, M. and Iida, K. "End -toEnd Header Compression over Software-Defined Networks: A Low Latency Network Architecture," in 2012 4th International Conference on Intelligent Networking and Collaborative Systems (INCoS), pp. 493-494, 2012

[20] Kassler, A., Skorin-Kapov, L., Dobrijevic, O. Matijasevic, M. and Dely, P. "Towards QoE-driven multimedia service negotiation and path optimization with software defined networking," 20th International Conference on Software, Telecommunications and Computer Networks (SoftCOM), pp. 1-5, 2012.

[21] Nabaei, A., Hamian, M., Parsaei, M. R., Safdari, R., Samad-Soltani, T., Zarrabi, H. and Ghassemi, A. "Topologies and performance of intelligent algorithms: a comprehensive review," Artificial Intelligence Review, pp. 1-25, 2016. doi:10.1007/s10462-016-9517-3 